\title{
Physical device modeling of Si/Si1- xGex multi-quantum well detector to optimize Ge content for higher thermal sensitivity
}

Atia Shafique, Shahbaz Abbasi, Omer Ceylan, Canan B. Kaynak, Mehmet Kaynak, et al.

Atia Shafique, Shahbaz Abbasi, Omer Ceylan, Canan B. Kaynak, Mehmet Kaynak, Yasar Gurbuz, "Physical device modeling of Si/Si1-xGex multiquantum well detector to optimize Ge content for higher thermal sensitivity," Proc. SPIE 10624, Infrared Technology and Applications XLIV, 106241A (29 May 2018); doi: $10.1117 / 12.2305003$

SPIE. Event: SPIE Defense + Security, 2018, Orlando, Florida, United States 


\title{
Physical device modeling of $\mathrm{Si} / \mathrm{Si}_{1-x} \mathrm{Ge}_{x}$ multi-quantum well detector to optimize Ge content for higher thermal sensitivity
}

\author{
Atia Shafique $^{\mathrm{a}}$, Shahbaz Abbasi ${ }^{\mathrm{a}}$, Omer Ceylan ${ }^{\mathrm{a}}$, Canan B. Kaynak ${ }^{\mathrm{b}}$, Mehmet Kaynak ${ }^{\mathrm{a}, \mathrm{b}}$, and \\ Yasar Gurbuz ${ }^{\text {,* }}$ \\ aSabanci University, Orta Mahalle, Tuzla 34956 Istanbul, Turkey \\ ${ }^{\mathrm{b}}$ IHP, Im Technologiepark 25, 15236 Frankfurt (Oder), Germany
}

\begin{abstract}
This paper presents the physical device modeling of a $\mathrm{Si}_{\mathrm{Si}} \mathrm{Si}_{1-x} \mathrm{Ge}_{x}$ multi-quantum well (MQW) detector to optimize the Ge content in the $\mathrm{Si}_{1-x} \mathrm{Ge}_{x}$ well required to enhance thermal sensitivity for a potential microbolometer application. The modeling approach comprises a self-consistent coupled Poisson-Schroedinger solution in series with the thermionic emission theory at the $\mathrm{Si} / \mathrm{Si}_{1-x} \mathrm{Ge}_{x}$ heterointerface and quantum confinement within the $\mathrm{Si}_{1-x} \mathrm{Ge}_{x} \mathrm{MQW}$. The integrated simulation environment developed in Sentauruas WorkBench (SWB) TCAD is employed to investigate the transfer characteristics of the device consisting three stacks of $\mathrm{Si}_{1-x} \mathrm{Ge}_{x}$ wells with an active area of $17 \mu \mathrm{m} \times 17 \mu \mathrm{m}$ were investigated and compared with experiment data.
\end{abstract}

Keywords: Infrared imaging, microbolometer, $\mathrm{Si}_{1-x} \mathrm{Ge}_{x}$ multi-quantum well, Ge content, technology computer aided-design, temperature coefficient of resistance

\section{INTRODUCTION}

Infrared (IR) imaging systems are ubiquitously extending their application beyond the military realm into mainstream instruments in various domains such as industrial process control, thermography (predictive maintenance, building inspection), medical imaging, automotive safety and consumer electronic. ${ }^{1}$ IR imaging systems have evolved into very portable, easy to use and reasonably priced instruments. The thermal imaging systems mainly comprises the detector to convert the IR radiations to an electrical signal and read-out integrated circuit (ROIC) to improvise the further signal processing for ultimate video output. From technology perspective the uncooled technology has become an excellent alternative to the expensive cooled system for many commercial and industrial purposes. ${ }^{2}$ As they do not require any external cooling unit, they offer exceptional benefits in maintainability as well as significant reduction in size, complexity, and cost. ${ }^{3}$

In an uncooled system, the incident IR radiations are absorbed by thermally isolated detector resulting a change in temperature of the detector, followed by a change in the electrical parameters through various sensing mechanisms owing to the temperature variation. The thermistor-based microbolometer sensing principle rely on a change in the electrical resistance $(R)$ of a detector caused by the change in temperature due absorbed IR radiation. ${ }^{4}$ The tremendous efforts to perpetual research for new materials and innovative devices has steered up to meet the industry standards concerning both higher thermal sensitivity and lower noise. The thermal sensitivity of detector quantified by the temperature coefficient of resistance $(T C R)$ where the high $T C R$ implies a pronounced change in resistance for even small corresponding temperature change. The wide variety of commercially available resistive microbolometers consist of vanadium oxide $\left(\mathrm{VO}_{x}\right)^{5}$ and amorphous silicon detectors with $T C R$ in the range of $2-3 \%{ }^{6,7}$ Since the fabrication of $\mathrm{VO}_{x}$ is not compatible with generic Si process flow and requires peculiar processing steps thus necessitates finding alternative materials for a microbolometer. The development of low resistance a-Si/a-SiGe thin films seems to be an attractive choice as microbolometer material to enhance $T C R$ but with limited noise performance. $\mathrm{Si} / \mathrm{Si}_{1-x} \mathrm{Ge}_{x}$ multi-quantum well (MQW) has been proposed as a promising solution for microbolometers ${ }^{8,9}$ because of enhanced thermal sensitivity and superior noise performance owing to ease of bandgap tailoring as the Ge content is increased in an epitaxially grown $\mathrm{Si}_{1-x} \mathrm{Ge}_{x}$ alloy.

Further author information: E-mail: yasar@sabanciuni.edu, Telephone: +90 (216) 483-9533 


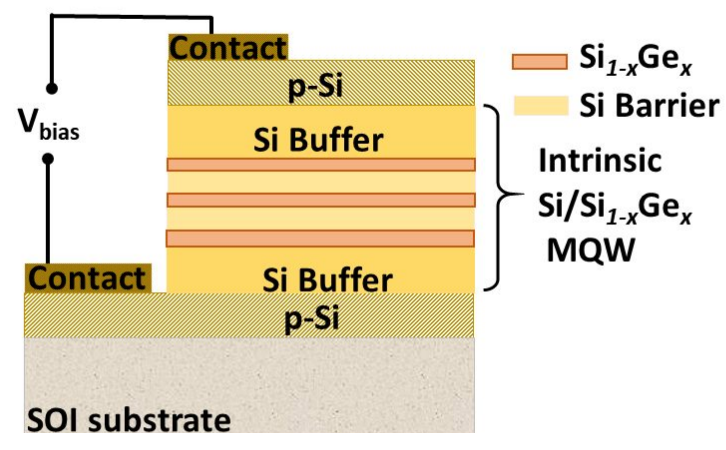

Figure 1. Cross-sectional view of $\mathrm{Si} / \mathrm{Si}_{1-x} \mathrm{Ge}_{x} \mathrm{MQW}$ device structure.

\section{DEVICE STRUCTURE AND SIMULATION FRAMEWORK}

A device structure formed by alternating layers of intrinsic $\mathrm{Si}$ and $\mathrm{Si}_{1-x} \mathrm{Ge}_{x}$ as shown in Figure 1, forming quantum well in SiGe layers for the holes. ${ }^{10}$ The thermal excitation caused by absorption of the IR radiation initiates emission of the holes from the SiGe wells into the Si followed the carrier transport in the direction of electric field due to applied bias (Vbias).

The integrated simulation framework in Sentaurus WorkBench (SWB) TCAD comprises the 2-D geometry of the structure ${ }^{11}$ and the doping profile along with the refined mesh definition in the sentaurus device editor. The device transfer characteristics (I-V) were obtained by the coupled self-consistent solution of Drift-diffusion formulation in series thermionic emission and Poisson-Schroedinger solver. Furthermore, the Massetti mobility model, bandgap narrowing, and carrier recombination (ShockleyReadHall and Auger) were also included for numerical simulation. The valence band offsets and doping profiles need to be optimized as a function of $\mathrm{Ge}$ mole fraction $x$ in $\mathrm{Si}_{1-x} \mathrm{Ge}_{x}$ MQW. In order to match the transfer characteristics obtained from simulation to the measurement requires optimization and fine tunning of the valence band offset $\left(\Delta E_{V}\right)$ and doping profile depending on $x$. Figure 2-a shows the optimized profile verified by SIMS analysis employed for for $\mathrm{Si} / \mathrm{Si}_{0.5} \mathrm{Ge}_{0.5}$ MQW simulation. Figure 2-b shows the band discontinuity formed in the heterostructure for the given profile in Figure 2-a with substantial valence bandoffset.

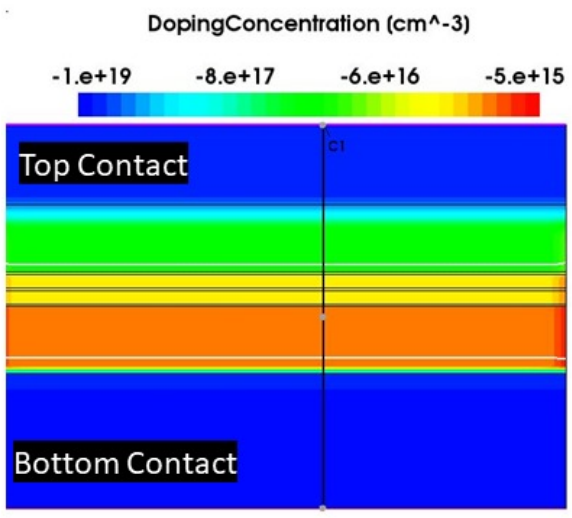

(a)

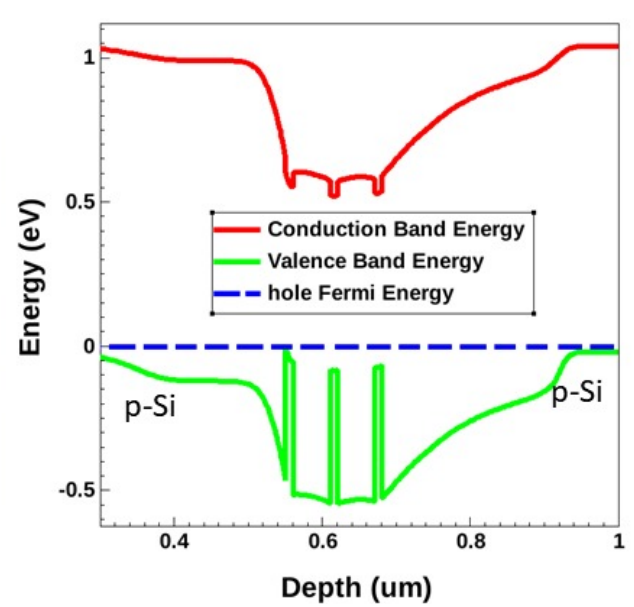

(b)

Figure 2. (a) The optimum doping profile used for $\mathrm{Si} / \mathrm{Si}_{0.5} \mathrm{Ge}_{0.5} \mathrm{MQW}$ simulation (b) Corresponding energy band diagram under equilibrium at $298 \mathrm{~K}$. 


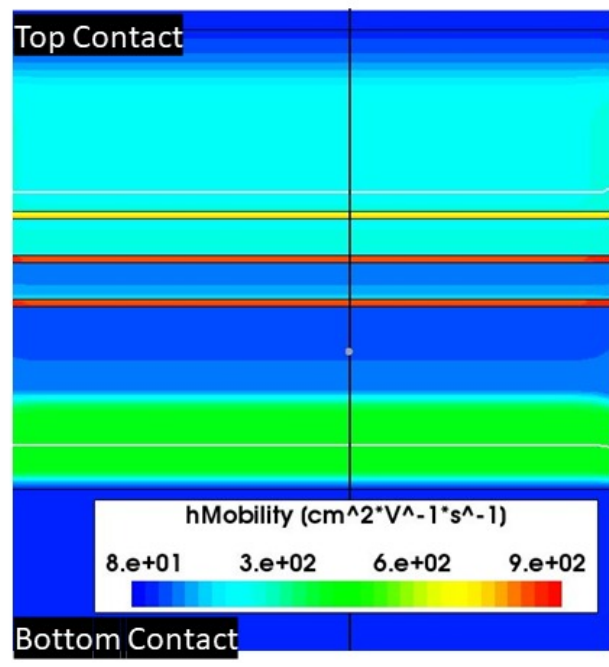

(a)

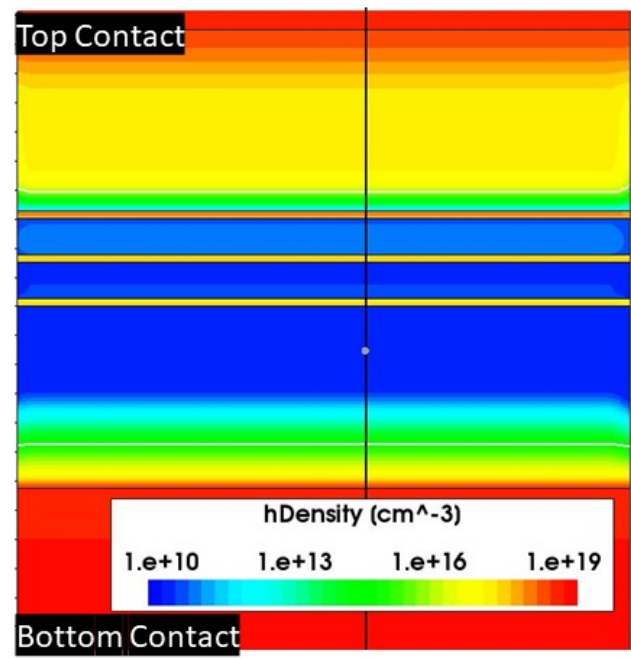

(b)

Figure 3. (a) Doping and temperature dependent Hole mobility and, (b) Net hole density estimates in two dimensional $\mathrm{Si} / \mathrm{Si}_{0.5} \mathrm{Ge}_{0.5} \mathrm{MQW}$ structure obtained from the simulation at $298 \mathrm{~K}$.

The doping dependent hole mobility in the various regions of device structure extracted from simulations presented in Figure 3-a indicates that the hole mobility is significantly higher in the SiGe wells. The net hole density shown in Figure 3-b is is determined by coupled Poisson-Drift diffusion solution by in the bulk Si regions whereas quantization model is used to compute hole density in the each SiGe well individually.

\section{SIMULATION AND EXPERIMENT COMPARISON}

For a quantitative comparison between simulation and experiment, the bandgap $\left(E_{g}\right)$ have been adjusted and fine tunned as a function of given Ge mole fraction $(x)$ as well as the doping profile have adjusted in a rigorous manner.

Simulated and measured I-V are fairly matched over the bias range as plotted in Figure 4-a, except some degree of deviation at very low Vbias, which can be caused due to high contact resistance in the actual device. The nonlinearity in I-V characteristics is considered as inherent property of the MQW structure. ${ }^{12}$ The net current density through the device increases for elevated temperature manifesting the thermal excitation of large number of carriers surmounting the barrier between Si and the SiGe well. Subsequently, the DC-resistance $R$ decreases over the temperature range $278 \mathrm{~K}-323 \mathrm{~K}$ as shown in Figure 4-b,implies that the temperature coefficient of resistance $(T C R)$ is negative as in general for the semiconductor materials. The measurement data was obtained from various test devices located at different sites on the wafer. Figure 4-b shows explicit data consistency and uniformity of device processing and fabrication for various data sets (A, B, C, D, E, F) ${ }^{11}$

The resistance $R(T)$ is defined as a function of temperature $T$, expressed by $(1)^{8}$

$$
R(T)=R_{o} \exp \frac{E_{a}}{k_{B} T}
$$

where $E_{a}$ is the activation energy which defines the effective barrier height owing to $\Delta E_{V}$.

$$
E_{a}=E_{F, p}-E_{V}
$$

$E_{F, p}$ is hole fermi energy, $E_{V}$ is the valence band edge of the surrounding Si barrier. TCR is defined as the rate of change of resistance w.r.t temperature (3) which can be determined by taking the natural logarithm of (1), 


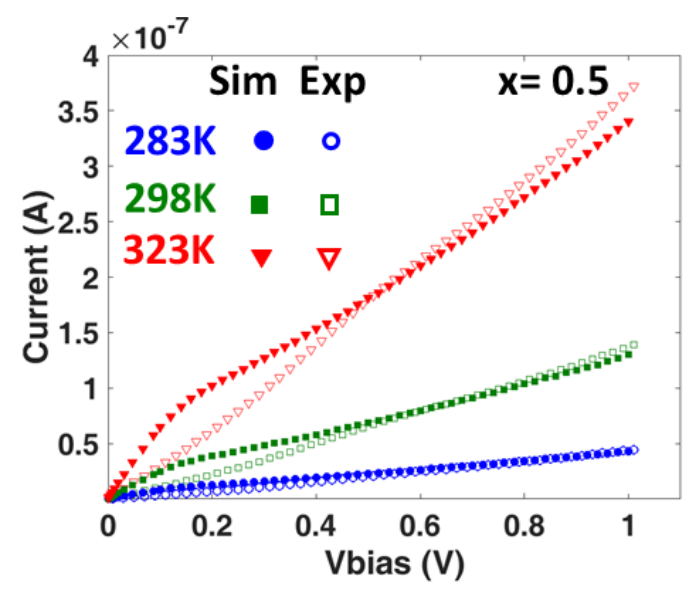

(a)

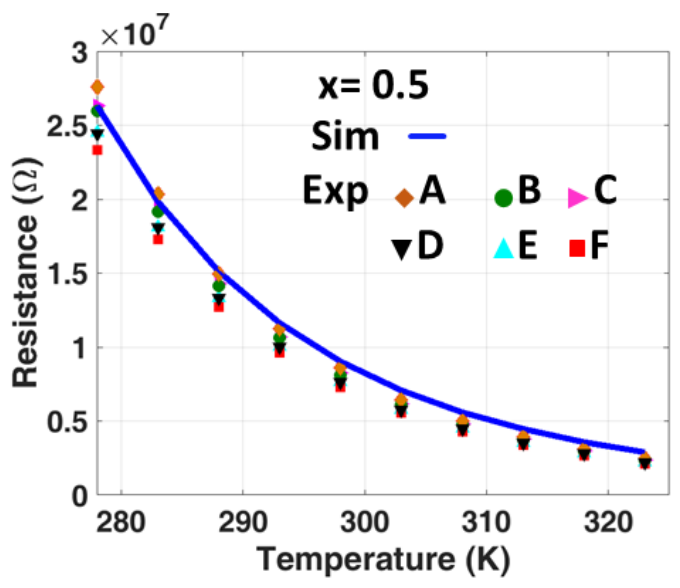

(b)

Figure 4. The simulated results compared with experimental data for $\mathrm{Si} / \mathrm{Si}_{0.5} \mathrm{Ge}_{0.5} \mathrm{MQW}$ device (a) Transfer characteristics (I-V) (b) Variation of $R$ over the temperature range of $278 \mathrm{~K}-323 \mathrm{~K}$ at fixed Vbias $=0.3 \mathrm{~V}$ plotted against experiment data obtained from various test devices (A, B, C, D, E, F) at different locations over the wafer.

and then computing partial derivative w.r.t temperature $T$, stated by (4):

$$
\begin{gathered}
T C R=\frac{1}{R} \frac{\partial R(T)}{\partial T} \\
T C R=\frac{1}{R} \frac{\partial R(T)}{\partial T}=-\frac{E_{a}}{k_{B} T_{r e f}^{2}}
\end{gathered}
$$

$k_{B}$ is the Boltzmann's constant and $T_{r e f}$ is the temperature at which the TCR is calculated.

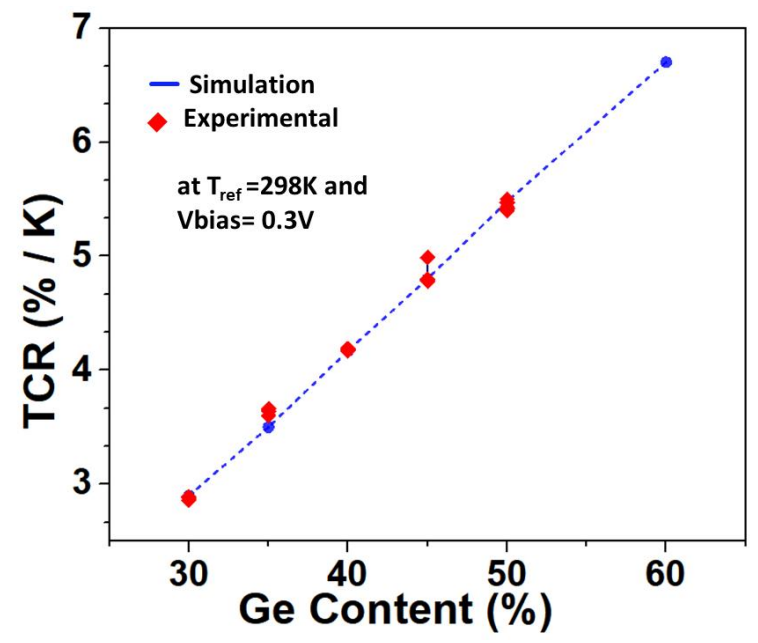

Figure 5. Comparison of simulated and measured $T C R$ for various the Ge mole fraction in $\mathrm{Si}_{1-x} \mathrm{Ge}_{x} \mathrm{MQW}_{\mathrm{device}}$.

Equation (4) explicitly shows that the thermal sensitivity can be enhanced with the higher $E_{a}$, which implies the larger barrier height (2) owing to larger $\Delta E_{V}$. In the case of $\mathrm{Si} / \mathrm{Si}_{1-x} \mathrm{Ge}_{x} \mathrm{MQW}$ the barrier height is linearlydependent on the Ge mole fraction $(x)$ present in the $\mathrm{Si}_{1-x} \mathrm{Ge}_{x}$ alloy constrained by the critical thickness criteria 
for the strained alloy. $E_{a}$ extracted by determining the slope $E_{a} / k_{B}$ of the Arrhenius plot is used to calculate $\mathrm{TCR}$ at $T_{r e f}=298 \mathrm{~K}$ for the various amounts of Ge mole fraction is presented and verified by the experimental data in Figure 5. Nevertheless, $T C R$ enhancement by increasing the barrier height comes at the the expense of larger $R$ as expressed by (1), $R$ increases exponentially with $E_{a}$. The simulation as well as measurement shows the intrinsic $\mathrm{Si} / \mathrm{Si}_{0.5} \mathrm{Ge}_{0.5} \mathrm{MQW}$ exhibits a very larger resistance of $8 \mathrm{M} \Omega$ at Vbais $=0.3 \mathrm{~V}$ and $\mathrm{T}=298 \mathrm{~K}$ from the plot in Figure 4-b.

Noise is considered an equally important parameter as $T C R$ when defining the Figure-of-Merits for any detector. In particular, the flicker noise $1 / \mathrm{f}$ dominates at low frequencies $(1 \mathrm{~Hz} \sim 30 \mathrm{~Hz})$ whereas the Johnson Noise $(4 k T R)$ dominates at higher frequencies and primarily contributes to the noise floor over the useful bandwidth. ${ }^{12}$ Inevitably, a detector with larger $R$ exhibits higher Johnson Noise, as well as endures a great deal of challenge for a readout design. ${ }^{13}$ As a matter of fact, (1) and (4) renders the critical design constraint and trade-off in terms of $T C R$ and $R$. From a detector point of view, in order to improve the over all Signal-to Noise ratio, the Ge content in a well must be optimized in a way to improve $T C R$ whilst keeping $R$ at moderate value. For a $\mathrm{Si}_{1-x} \mathrm{Ge}_{x}$ MQW device, $R$ can be reduced by selectively and carefully optimized doping in the MQW regions such that $E_{F, p}$ shifts close to the valence band edge of the well without losing the effective barrier height and thus $T C R$ is not deteriorated.

\section{CONCLUSIONS}

This paper presents a critical design trade-off expressed in terms of $T C R$ and $R$ in a $\mathrm{Si}_{1} / \mathrm{Si}_{1-x} \mathrm{Ge}_{x}$ multi quantum well structure as a function of Ge mole fraction $(x)$ which ultimately defines a performance metric for a resistive microbolometer. The integrated simulation framework was developed to study the key physical processes and their interactions governing the overall device transfer characteristics. The simulation shows a linear increase in $T C R$ as a function of Ge mole fraction $(x)$ but at the cost of a large resistance due to enlarge barrier height which limits the current transport. Nevertheless, a larger $R$ degrades the overall noise performance. The experimental data verifies the simulation results. An optimized value of $x$ should be considered for a $\mathrm{Si} / \mathrm{Si}_{1-x} \mathrm{Ge}_{x} \mathrm{MQW}$ detector augmenting the overall performance metric of a detector.

\section{ACKNOWLEDGMENTS}

This work was financially supported by the Scientific and Technological Research Council of Turkey under Project Grant 115E098.

\section{REFERENCES}

[1] Mounier, E., "Uncooled infrared imagers market and technology trends," tech. rep., Yole Development (2017).

[2] Kruse, P. W., [Uncooled Thermal Imaging Arrays, Systems, and Applications], SPIE PRESS BOOK (July 2001).

[3] Rogalski, A., "Infrared detectors for the future," Acta Physica Polonica A 116, 389-406 (sep 2009).

[4] Niklaus, F., Vieider, C., and Jakobsen, H., "MEMS-based uncooled infrared bolometer arrays: a review," in [MEMS/MOEMS Technologies and Applications III], Chiao, J.-C., Chen, X., Zhou, Z., and Li, X., eds., SPIE (nov 2007).

[5] Li, C., "Overview of DRS uncooled VOx infrared detector development," Optical Engineering 50, 061017 (jun 2011).

[6] Tissot, J., Trouilleau, C., Fieque, B., Crastes, A., and Legras, O., "Uncooled microbolometer detector: recent developments at ULIS," Opto-Electronics Review 14 (jan 2006).

[7] Yon, J.-J., Mottin, E., and Tissot, J.-L., "Latest amorphous silicon microbolometer developments at LETILIR," in [Infrared Technology and Applications XXXIV], Andresen, B. F., Fulop, G. F., and Norton, P. R., eds., SPIE (apr 2008). 
[8] Forsberg, F., Lapadatu, A., Kittilsland, G., Martinsen, S., Roxhed, N., Fischer, A. C., Stemme, G., Samel, B., Ericsson, P., Hoivik, N., Bakke, T., Bring, M., Kvisteroy, T., Ror, A., and Niklaus, F., "CMOSintegrated $\mathrm{Si} / \mathrm{SiGe}$ quantum-well infrared microbolometer focal plane arrays manufactured with very largescale heterogeneous 3D integration," IEEE Journal of Selected Topics in Quantum Electronics 21, 30-40 (jul 2015).

[9] Jiang, B., Dong, T., Su, Y., He, Y., and Wang, K., "Epitaxial growth and characterization of self-doping $\mathrm{Si}_{1-x} \mathrm{Ge}_{x} / \mathrm{Si}$ multi-quantum well materials," Journal of Microelectromechanical Systems 23, 213-219 (feb 2014).

[10] Shafique, A., Durmaz, E. C., Cetindogan, B., Yazici, M., Kaynak, M., Kaynak, C. B., and Gurbuz, Y., "Design of monocrystalline Si/SiGe multi-quantum well microbolometer detector for infrared imaging systems," Proc.SPIE 9819 (2016).

[11] Kaynak, C. B., Yamamoto, Y., Goritz, A., Korndorfer, F., Zaumseil, P., Kulse, P., Schulz, K., Wietstruck, M., Shafique, A., Gurbuz, Y., Kaynak, M., and Kaynak, M., "High performance thermistor based on si1xGex /si multi quantum wells," IEEE Electron Device Letters (2018, early access).

[12] Ericsson, P., Hglund, L., Samel, B., Savage, S., Wissmar, S., berg, O., Kllhammer, J.-E., and Eriksson, D., "Design and evaluation of a quantum-well-based resistive far-infrared bolometer," in [Electro-Optical and Infrared Systems: Technology and Applications VII], Huckridge, D. A. and Ebert, R. R., eds., SPIE (oct 2010).

[13] Galioglu, A., Abbasi, S., Shafique, A., Ceylan, O., Yazici, M., Kaynak, M., Durmaz, E. C., Arsoy, E. G., and Gurbuz, Y., "A low-power cmos readout IC design for bolometer applications," Proc.SPIE 10177 (2017). 